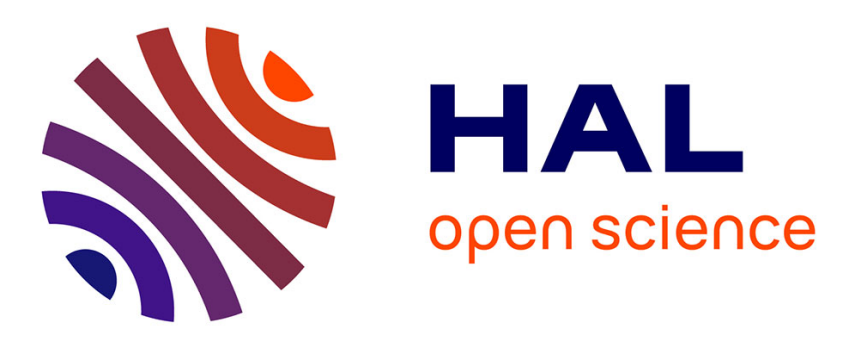

\title{
Influence of the Magnetic Field Nonuniformity on an X-Band Microstrip Y-Junction Circulator Bandwidth: Theory/Experiment Comparison
}

Annaïg Guennou, Bernard Della, Patrick Queffelec, Philippe Gelin, Jean-Luc Mattei

\section{To cite this version:}

Annaïg Guennou, Bernard Della, Patrick Queffelec, Philippe Gelin, Jean-Luc Mattei. Influence of the Magnetic Field Nonuniformity on an X-Band Microstrip Y-Junction Circulator Bandwidth: Theory/Experiment Comparison. IEEE Transactions on Magnetics, 2007, 43 (6), pp.2642. 10.1109/TMAG.2007.893785 . hal-00172026

\section{HAL Id: hal-00172026 https://hal.science/hal-00172026}

Submitted on 13 Sep 2007

HAL is a multi-disciplinary open access archive for the deposit and dissemination of scientific research documents, whether they are published or not. The documents may come from teaching and research institutions in France or abroad, or from public or private research centers.
L'archive ouverte pluridisciplinaire HAL, est destinée au dépôt et à la diffusion de documents scientifiques de niveau recherche, publiés ou non, émanant des établissements d'enseignement et de recherche français ou étrangers, des laboratoires publics ou privés. 


\title{
Influence of the Magnetic Field Nonuniformity on an X-Band Microstrip Y-Junction Circulator Bandwidth: Theory/Experiment Comparison
}

\author{
A. Guennou, B. Della, P. Quéffélec, P. Gelin, and J. L. Mattei \\ LEST, UMR CNRS 6165, CS 93837, 29238 Brest cedex 3, France
}

\begin{abstract}
We propose a theoretical approach aimed at accurately describing the electromagnetic (EM) response of a microstrip Y-junction circulator. This analysis is predictive since it relies on the insertion of both the nonuniformity of the static field of polarization and the ferrite material magnetization state. We fabricated proof-of-concepts to compare the responses issued from two available EM analyses, namely our approach and the HFSS software one. The key-role of a rigorous modeling of the microstrip Y-junction circulator is highlighted through a comparison of simulation data and experimental results.
\end{abstract}

Index Terms-Ferrite circulators, magnetic field effects.

\section{INTRODUCTION}

$\mathbf{T}$ HE RAPID growth of needs in communication systems for civil or military applications requires a strong optimization of the microwave devices. In this context, small size circuits with high operating frequencies and produced at low cost become a must. A key component integrated in transmit/receive modules is the circulator.

In X-band, the device the most commonly used in the microwave systems is the Y-junction circulator. It can be found as a $6.25 \mathrm{~mm} \times 6.25 \mathrm{~mm} \times 6$-mm-sized device with a $5.6 \%$-bandwidth centered at $9 \mathrm{GHz}$ and insertion losses lower than $0.5 \mathrm{~dB}$. However, until now it has not fully met the criteria required by the new applications in the telecommunication market, i.e., miniaturization, low production cost and operating frequencies in the millimeter wave range. To fulfill the previous schedule of conditions while displaying good electromagnetic performances, the design and realization of circulators have to be based on new materials (hexaferrites, magnetoelectric composites), new technological processes (LTCC, spin coating, skillscreen printing technique) and on the use of predictive theoretical tools. Our aim is to propose an accurate electromagnetic analysis (EMA) of Y-junction circulators enabling a better understanding of the physical phenomena appearing in various configurations (conventional saturated YIG-based circulators [1], self-biased hexaferrite structures [2]) in order to optimize their performances.

One among the major parameters that strongly affect the performances of circulators is the dc-bias field. Some studies have shown a reduction of the Y-junction circulator transmission band induced by the nonuniformity of the dc-bias field created, within the ferrite slab, by permanent magnet [3]. In practice, this nonuniformity is quite frequent because the shape of the ferrite sample is rarely an ellipsoid [4], [5], and the size of the polarization circuit has to be strongly reduced for miniaturization purpose. The increasing needs for miniature planar microwave circuits have encouraged designers to employ microstrip technology because of available solutions liable to enhance circulators compactness. The objective would be to

Digital Object Identifier 10.1109/TMAG.2007.893785 polarize the device with only one magnet. However, compared to the stripline structure this topology enhances the nonuniformity of the magnetic dc-bias field, but at the expense of device performances.

How et al. have observed theoretically and experimentally the apparition of a cutoff zone in the device bandwidth [3]. Together, these considerations highlight the need for thorough investigations targeted to the modeling of microstrip Y-junction circulator. Indeed, it is paramount in such studies to take into account, at the same time, the field nonuniformity, the unsaturated state of certain regions of the ferrite puck, and the access lines on dielectric or ferrite substrate.

To evidence how the dc-bias field affects the device response, we fabricated an X-band microstrip circulator. The scattering parameters (S-parameters) obtained from simulations enabled us to determine which of HFSS and our theoretical approach provides the response closer to the one experimentally produced by our in-lab-made circulator.

\section{THEORY}

The operation of Y-junction circulator was first described by Bosma (1962), whose analysis rested on several assumptions. At first, he assumed a uniform internal field for the circulator constitutive ferrite, which is not the case in practice. Some demagnetizing fields appear at the periphery of the ferrite slab since the puck is not an ellipsoid. Moreover, the dc-bias field created by the magnet is also nonuniform. The magnitude of the dc-bias field is, thus, insufficient for medium saturation in certain regions of the ferrite puck. Bosma made a second assumption about the saturation of the magnetic matter. He used the Polder formulations to compute the permeability tensor appearing in the Maxwell equations. As previously mentioned, the nonuniformity of the internal field in the ferrite prevents some regions from being saturated. Other formulations of the permeability tensor are necessary to accurately predict the dynamic response of the ferrite medium.

Thus, a thorough calculation of the device S-parameters requires the modification of conventional EMA. It led us to include, in our EMA, a spatial variation of the dc-bias field and a suitable effective permeability tensor. The latter takes into account the real magnetized state of the different regions of the ferrite sample [6]. To consider the variation of the internal field, the ferrite junction is divided into several annuli [7]. Each of 
them can be characterized by its own uniform local internal field, computed from a magnetostatic analysis (Maxwell 3-D), as well as its own local permeability tensor obtained from the model described in [6].

The solution of the Maxwell equations gives the EM field for each annulus. The field in the inner disk constitutes the general solution given by Bosma. The application of boundary conditions at each interface enabled us to get the expression of the EM field at the periphery of the ferrite disc and to calculate the S-parameters. We wrote a computer code to calculate the $\mathrm{S}$-parameters of the circulator for a uniformly or nonuniformly biased structure. To validate our theoretical tool, we compared our results of calculation with those of earlier calculations by others [3]. It showed a good agreement between our approach and those reported in the literature for either a uniform or a nonuniform field distribution [8]. Our EMA highlighted a cutoff band due to a bias field nonuniformity, eventually responsible for a total degradation of the device response.

As previously mentioned, the microstrip technology offers interesting prospects compared to the stripline structure. However, the microstrip approach implies a stronger nonuniformity of the dc-bias field. In order to study more accurately the effect of field nonuniformity upon the device response, we fabricated an X-band-operating microstrip circulator.

\section{RESULTS}

The fabricated device under study is of a large size, and the properties of the ferrite are not optimal. It is worth underlining that our goal was not the realization of an optimal structure in terms of performances or size; it was rather to evidence and gain insight into the problems liable to occur in this structure in order to further predict them through an EMA more predictive than those available in the literature or implemented in commercial simulators.

The 508- $\mu \mathrm{m}$-thick ferrite slab used to fabricate the circulator is a yttrium iron garnet (YIG: $4 \pi M_{S}=1760 \mathrm{G}, \Delta H=85$ Oe, $\varepsilon_{f}=15$ ). Its other dimensions are $25.4 \mathrm{~mm} \times 25.4 \mathrm{~mm}$. The circulator ground plane, conductive strips and junction are obtained by depositing a $4-\mu \mathrm{m}$-thick layer of gold. The junction radius is $2 \mathrm{~mm}$. The access lines (length $=1.95 \mathrm{~mm}$, width $=$ $1 \mathrm{~mm}$ ) are deposited on the ferrite substrate and connected to a $50-\Omega$ line.

The dc-bias field is only applied to the junction normal to the ferrite slab. It is provided either by a samarium-cobalt ( $\mathrm{Sm}-\mathrm{Co}$ ) magnet or two Sm-Co magnets (above and below the junction). The radius of each magnet is $2 \mathrm{~mm}$. We used several configurations of magnets to highlight the influence of the dc-bias field nonuniformity on the device response; it showed us that the dc-bias field is more uniform and more unidirectional when the junction is polarized by two magnets. However, even with a two-magnet configuration, the nonuniformity of the internal field still exists. On the other hand, using only one magnet would be more appropriate for miniaturization purpose. However, in this case, the field intensity is low with directional changes, in particular, on the magnet edges. Moreover, this configuration enhances the field nonuniformity in the junction. These different configurations allow one to consider various spatial variations of the internal field in the junction region. The applied field mea-

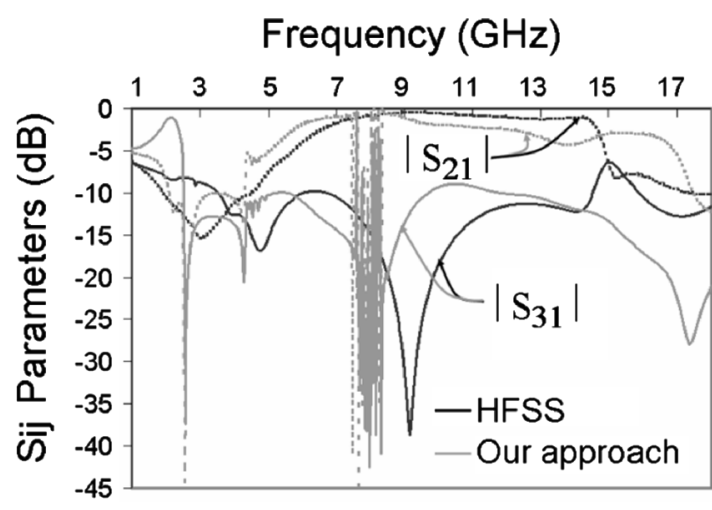

Fig. 1. Comparison (HFSS/Our approach) of the calculated response of the circulator biased nonuniformly by one magnet.

sured in the junction center was about 3000 Oe in each configuration. To obtain the same strength at the center, the magnets were offset from the ferrite slab with foam slabs $\left(\varepsilon_{r}=1.07\right)$ of different thicknesses.

A magnetostatic analysis (Maxwell 3-D) gave us the spatial variation of the applied field and, thus, the spatial variation of the internal field in the ferrite junction. The latter was further introduced in our EMA and in the HFSS software to get a realistic response of the device. Fig. 1 presents the S-parameters obtained from each analysis. Our approach evidences a cutoff zone in the device response at $8.5 \mathrm{GHz}$ and below, which is unseen in the HFSS simulation curve.

How et al. attributed these singularities to the dc-bias field nonuniformity [3]. To locate the cutoff bands in the circulator response, the propagation constant in saturated ferrite, $k_{\mathrm{eff}}$, has to be thoroughly studied. By using the Polder formulation, $k_{\mathrm{eff}}$ can be expressed as a function of the nonuniform internal field, $H_{i}(r)$, the saturation magnetization, $4 \pi M_{s}$, and frequency, $f$

$$
\begin{aligned}
k_{\mathrm{eff}}^{2}(r) & =\left(\frac{2 \pi f}{c}\right)^{2} \\
& \frac{\left[4 \pi M_{S}+H_{i}(r)+f / \gamma\right] \cdot\left[4 \pi M_{S}+H_{i}(r)-f / \gamma\right]}{H_{i}(r)\left[4 \pi M_{S}+H_{i}(r)\right]-(f / \gamma)^{2}}
\end{aligned}
$$

where $\gamma$ is the gyromagnetic ratio $(\gamma=2.8 \mathrm{MHz} / \mathrm{Oe})$, and $r$ is the radius in the junction.

By setting the numerator and denominator of this expression to 0 , How et al. found two frequencies characteristic because of the lack of propagation.

$$
\left\{\begin{array}{l}
f_{1}(r)=\gamma\left\{H_{i}(r)\left[H_{i}(r)+4 \pi M_{S}\right]\right\}^{1 / 2} \\
f_{2}(r)=\gamma\left[H_{i}(r)+4 \pi M_{S}\right] .
\end{array}\right.
$$

As the internal field strength $H_{i}(r)$ found in (2) is not unique in the ferrite junction, $f_{1}$ and $f_{2}$ likely correspond to two cutoff bands $\Delta f_{1}$ and $\Delta f_{2}$. Our theoretical approach predicted two cutoff bands in the circulator response (Fig. 1). Their values are in good agreement with (2) when the dc-bias field strength variation given by Maxwell 3-D is kept for the calculation of $\Delta f_{1}$ and $\Delta f_{2}$.

It is worth noting that the simulated S-parameters obtained from our EMA are modified whenever different ferrite thicknesses are considered. However, the peaks appearing in the device response are located at the same frequencies. Indeed, the 


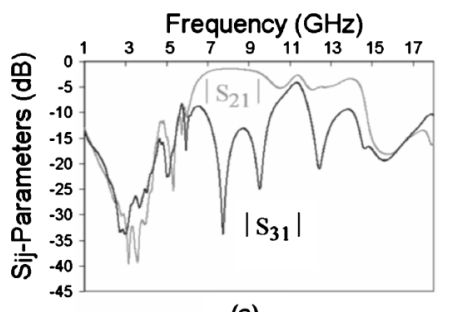

(a)

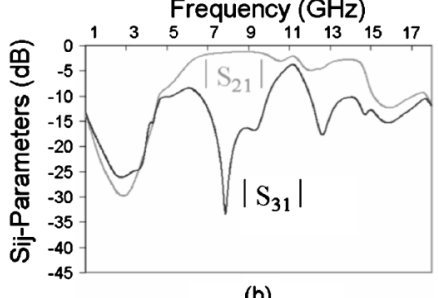

(b)
Fig. 2. Measured S-parameters of the circulator biased (a) by one Sm-Co magnet (thickness $=4 \mathrm{~mm}$ ) placed at $0.75 \mathrm{~mm}$ above the ferrite disk, (b) by two Sm-Co magnets (thickness $=2 \mathrm{~mm}$ ) respectively placed at $1 \mathrm{~mm}$ above and below the ferrite disk.

singular frequencies are connected to the junction internal field magnitudes and to the sample saturation magnetization. So, in our study, the ferrite thickness does not influence the localization of the peaks.

Now, let us compare the two theoretical responses (Fig. 1) to the experimental one. The S-parameters of the fabricated device were measured with an Agilent E8364A vector network analyzer. Fig. 2 shows the experimental response obtained for our microstrip Y-junction circulator biased by two different magnet configurations.

One should note that the center frequency, $f_{c}=9.2 \mathrm{GHz}$, given by HFSS (Fig. 1) is higher than the one measured with the two-magnet configuration $\left(f_{c}=7.9 \mathrm{GHz}\right)$ (Fig. 2), which is nearly the value, $f_{c}=8.1 \mathrm{GHz}$, predicted by our approach, (Fig. 1). Despite the introduction in HFSS software of a strong variation of dc-bias field strength, the peaks observed in the experimental response of the one-magnet-biased device do not appear in the simulator-produced S-parameters [Fig. 2(a)]. As expected, the circulator response is worsened when the junction is polarized by a single magnet [Fig. 2(a)]. However, the frequencies of the observed peaks disagree with the theoretical one given by (2) (the signal is degraded below $6.2 \mathrm{GHz}$ ). Indeed, the dc-bias field strength at the center of the junction is about 3000 Oe/ (Fig. 2); one should also note a cutoff band in signal transmission at about $8.5 \mathrm{GHz}$ and below this value (2). The signal is worsened at a frequency lower than the expected one, i.e., $6.2 \mathrm{GHz}$ [Fig. 2(a)], which corresponds to a dc-bias field strength of $2214 \mathrm{Oe}$; this made us wonder whether the field magnitudes at the center of the junction are responsible for device response degradation.

Our tool considers the substrate of the access line as isotropic, which is not the case in practice since a field-induced anisotropy due to the dc-fringing field appears in the ferrite substrate in the vicinity of access lines.

To explain the cutoff band observed below $6.2 \mathrm{GHz}$ [Fig. 2(a)], we thoroughly studied the internal field of the ferrite junction edges and access lines. Indeed, a low field goes along with a worsened response. The change in wave/matter interaction induces an excitation of magnetostatic waves. Depending on the direction of polarization of the internal magnetic field different types of magnetostatic waves can propagate. In our opinion, the singularities observed in the circulator response are induced by the propagation of magnetostatic volume or surface waves along the access lines. Thus, conducting a rigorous study of changes in the internal magnetic field at the origin of bias in the ferrite along the access line is a prerequisite to wave localization.

\section{CONCLUSION}

This study pointed out the need of predictive theoretical tools for an accurate determination of the microstrip Y-junction circulator performances. Indeed, the experimental response of our device showed a cutoff band, which was missing in the transmission signal obtained from HFSS software; but this cutoff band was predicted by our approach to lie within a higher frequency band.

It is essential to concomitantly consider the nonuniformity of fields, the unsaturated state in certain regions of the ferrite junction, the access lines on dielectric substrate or ferrite. This will drive us to modify our EMA in order to consider the case of access lines on anisotropic ferrite substrate liable to be magnetized in a direction different from the junction one. Circulators with access lines on dielectric substrate will be made to determine whether the frequency cutoff band appears or not.

\section{REFERENCES}

[1] H. Bosma, "On the stripline Y-circulation at UHF," IEEE Trans. Microw. Theory Tech., vol. MTT-12, no. 1, pp. 61-72, Jan. 1964.

[2] A. Guennou, P. Quéffélec, P. Gelin, and J. L. Mattei, "Self-biased Y-junction circulators using polycrystalline hexaferrite: An accurate electromagnetic analysis," J. Appl. Phys., vol. 99, 2006, 08P505.

[3] H. How, S. A. Oliver, S. W. McKnight, P. M. Zavracky, N. E. McGruer, C. Vittoria, and R. Schmidt, "Influence of nonuniform magnetic field on a ferrite junction circulator," IEEE Trans. Microw. Theory Tech., vol. 47, no. 10, pp. 1982-1989, Oct. 1999.

[4] R. I. Joseph and E. Schloemann, "Demagnetizing field in nonellipsoidal bodies," J. Appl. Phys., vol. 36, no. 5, pp. 1579-1593, May 1965.

[5] M. Pardavi-Horvath, "Microwave applications of soft ferrites," $J$. Magn. Magn. Mater., pp. 171-183, 2000, 215-216.

[6] P. Gelin, P. Quéffélec, and F. Le Pennec, "Effect of domain and grain shapes on the dynamic behavior of polycrystalline ferrites. Application to the initial permeability," J. Appl. Phys., vol. 28, $2005,053906$.

[7] C. M. Krowne and R. E. Neidert, "Theory and numerical calculations for radially inhomogeneous circular ferrite circulators," IEEE Trans. Microw. Theory Tech., vol. 44, no. 3, pp. 419-431, Mar. 1996.

[8] A. Guennou, P. Quéffélec, P. Gelin, and J. L. Mattei, "Coupled magnetostatic/electromagnetic studies of nonuniformly biased y-junction circulator: Application to transmission bandwidth increase," IEEE Trans. Magn., submitted for publication. fr).
Manuscript received October 31, 2006 (e-mail: annaig.guennou@univ-brest. 